\title{
Water-wise hand preparation - the true impact of our practice: A controlled before-and-after study
}

\author{
M S W Potgieter, ${ }^{1}$ MB ChB, FC Orth (SA), MMed (Orth) (SA); A Faisal, ${ }^{2}$ BS; A Ikram, ${ }^{1}$ MBBS, FC Orth (SA), \\ M C Burger, ${ }^{1}$ BSc, BMedSc Hons, MMedSc, $\mathrm{PhD}$ \\ ${ }^{1}$ Division of Orthopaedic Surgery, Department of Surgical Sciences, Faculty of Medicine and Health Sciences, Stellenbosch University, \\ Cape Town, South Africa \\ ${ }^{2}$ Department of Neurobiology, Physiology and Behavior, University of California, USA
}

Corresponding author: M C Burger(mcburger@sun.ac.za)

\begin{abstract}
Background. South Africa (SA) and other countries worldwide are experiencing extreme drought conditions. Since the start of the drought in SA, many ways of saving water have been proposed and innovative water-saving mechanisms have become part of the lives of communities. We investigated water use during surgical scrubbing procedures and possible interventions to reduce water consumption.

Objectives. To compare water use during surgical hand preparation before and after the implementation of specific water-saving interventions. Methods. This was a non-randomised controlled study, following a before-and-after design, of orthopaedic theatre personnel scrubbing for surgical cases at Tygerberg Hospital, Cape Town. A control (CON) group $(n=32)$ was established to observe standard practice for baseline measurements including total amount of water used, wash time and water flow rate during surgical hand preparation. After this, three interventions were randomly assigned to a single theatre each, where the same variables were measured. Intervention AS entailed using an alcohol scrub $(n=18)$, intervention SN $(n=12)$ had a dedicated assistant to open and close taps during scrubbing, and intervention SW $(n=12)$ made use of adjusted tap levers to allow the surgeon to open and close taps more easily. Analysis of variance was used to detect global differences between groups, and Tukey's post hoc test was performed to detect differences between groups.

Results. Significant differences in water use $(p<0.001)$, wash time $(p<0.001)$ and water flow rate $(p<0.001)$ were observed between the four groups. On average, the AS group used the least water per scrub (mean (standard deviation) 0.82 (1.43) L), which was significantly less than the CON $(5.56(1.79) \mathrm{L} ; p<0.001)$ and SN $(2.29(0.37) \mathrm{L} ; p=0.002)$ groups. The amount of time spent per scrub was significantly less in the AS group than all the other groups ( $p<0.05$ for all comparisons), with no significant differences observed between the CON, $\mathrm{SN}$ and SW groups independently. The SW group had the lowest mean water flow rate $(0.73(0.22) \mathrm{L} / \mathrm{min})$, which was significantly lower than the CON group $(2.19(0.84) \mathrm{L} / \mathrm{min} ; p<0.001)$. The flow rate of the $\mathrm{SN}$ group $(1.36(0.66) \mathrm{L} / \mathrm{min})$ was also significantly lower than that of the CON group $(p=0.005)$.

Conclusions. Water use during surgical hand preparation can easily be reduced by implementing easy and effective interventions. The practicality of interventions may differ between institutions, and their acceptance by surgical staff is important to ensure compliance. However, ensuring that alternative scrubbing options are available to surgical staff would equate to substantial savings over time.
\end{abstract}

S Afr Med J 2020;110(4):291-295. https://doi.org/10.7196/SAMJ.2020.v110i4.14044

Several parts of South Africa (SA) are currently experiencing severe drought conditions, and the drought that affected several provinces was declared a national disaster in February 2018. ${ }^{[1]}$ In the Western Cape Province water restrictions were implemented in several areas, whereby individuals were financially penalised for using too much water, while a water-shedding schedule was enforced in other areas. Dam levels in the province increased following winter rains, but water restrictions that urge each individual to consume no more than a set amount of water per day in the home and at the workplace remain in place.

Since the start of the drought, many ways of saving water have been proposed and innovative water-saving mechanisms have become part of the lives of many South Africans. These include immediate repair of water leaks, installation of grey-water recycling systems, and minimising water use for personal hygiene and the performing of daily tasks such as laundry or dishwashing. Saving water at the workplace is equally important, and the biggest area of water consumption in surgery is the water required for theatre use. It is obvious that some areas of water use in theatre cannot be compromised; these include autoclaving requirements and theatre cleaning. Although the use of disposable drapes can reduce the amount of water required for theatre preparation, surgical hand preparation is arguably one of the biggest potential ways of saving water over which the individual has control.

The current standard procedures for surgical hand preparation at Tygerberg Hospital (TBH) in Cape Town involve a 3 - 5-minute scrub with water and soap under a running tap before each surgical case. Although alcohol-based hand-scrub solutions are available for use and flexibility is allowed for the surgeon's preference, the method of choice for the majority of surgeons remains the traditional soapand-water technique. TBH has $\sim 20$ active theatres, each handling 5 - 10 surgical cases per day. The water used in surgical hand preparation at this hospital alone can therefore potentially reach a staggering amount. Modern theatre complexes may have basin setups that allow for user control of water flow, but unfortunately these systems are costly and not available in most SA government hospitals. The basin systems at TBH still have standard elbow taps, which means that while the surgeon is scrubbing, the tap is open and water flows freely. It is estimated that the average flow rate of an open tap is $15-18 \mathrm{~L} / \mathrm{min} \cdot{ }^{[2,3]}$ 
The practice of scrubbing with soap and water only once (before the first surgery of the day) and thereafter to use alcohol scrubs between patients is generally accepted, forms part of the World Health Organization (WHO) guidelines on hand hygiene in healthcare, and is recommended in areas where water sources are unreliable. ${ }^{[4]}$ Hubner et al. ${ }^{[5]}$ demonstrated the efficacy of hand disinfection with alcohol-based preparations only and recommended that a 15-second prewash with water and disinfectant soap be done once daily prior to starting clinical duties. Doing this will effectively reduce resident spores on the hands of the clinician. ${ }^{[5]}$ To date, several studies, including a Cochrane review, ${ }^{[6]}$ have come to similar conclusions. ${ }^{[7,8}$

\section{Objectives}

To compare water use during surgical hand preparation before and after specific water-saving interventions. The first objective was to quantify the amount of water used per day by measuring water consumption for scrubbing purposes in one division and extrapolating it to the hospital as a whole. Following this, we investigated potential water-saving mechanisms that: $(i)$ are easy and cost-effective to implement; (ii) provide adequate sterility for surgery; and (iii) are generally accepted methods of surgical hand preparation.

\section{Methods}

This non-randomised controlled study, which followed a controlled before-and-after design, was conducted at TBH. Prior to commencement of the study, ethical approval was obtained from the Stellenbosch University Health Research Ethics Committee (ref. no. N17/07/057) and institutional permission was obtained.

A baseline (control) group (CON) was established, after which three interventions were randomly assigned to a single theatre each, each with several procedures scheduled, on one day over a period of 12 hours.

\section{Baseline/control (CON) group}

Water consumption during scrubbing procedures of all surgical staff in the Division of Orthopaedic Surgery at TBH was observed over a period of 12 hours in order to establish a baseline of the standard water use per day in five orthopaedic surgery theatres. All surgeons were informed about the study and were urged not to change their behaviour even though their actions were being observed. Routine, standard-practice scrubbing procedures were used, which include the surgeon scrubbing his or her hands with $4 \%$ chlorhexidine gluconate soap (Bioscrub; B Braun Medical (Pty) Ltd, SA), using water. According to TBH policy, alcohol scrubs are also considered an acceptable method of scrubbing, and a $0.5 \%$ chlorhexidine and
$70 \%$ alcohol scrub (Biotane, B Braun Medical (Pty) Ltd) is available to surgeons who prefer this method of surgical hand preparation. Water use was measured by placing a container in the basin to catch all water, after which the total amount of water was weighed. One kilogram weight was estimated to equate to one litre of water. The amount of time spent scrubbing was also measured. In cases where surgeons made use of alcohol scrubs between surgeries, water use was reported as zero.

\section{Intervention groups}

Alcohol scrub intervention (AS)

As per WHO recommendations, surgeons completed a 15-second prewash with water and $4 \%$ chlorhexidine gluconate soap, after which a $0.5 \%$ chlorhexidine and $70 \%$ alcohol scrub was used. The alcohol scrub alone was used between surgeries. Total water use per surgeon per scrub was measured as previously described.

\section{Scrub nurse intervention (SN)}

A scrub nurse was stationed at each basin to assist the surgeons with opening/closing the elbow taps during scrubbing procedures. The tap was opened whenever the surgeon required water flow and closed when not in direct use. Total water use per surgeon per scrub was measured as previously described.

\section{Self-wash intervention (SW)}

Plumbers were asked to adjust tap levers to be perpendicular to the surgeon when in the off position (Fig. 1), allowing much easier elbow control of water flow rates during the scrubbing procedure. Surgeons and scrub nurses were asked to open taps only to rinse their hands and to close the taps while scrubbing. Total water use per surgeon per scrub was measured as previously described.

\section{Statistical analysis}

The main outcomes of the study, mean time per scrub (seconds/ scrub), mean litres of water per scrub and mean water flow $(\mathrm{L} / \mathrm{min})$, were calculated. All outcomes were normally distributed, so analysis of variance was used to determine whether there were any significant differences between the groups for each outcome. Where $p<0.05$, Tukey's post hoc test was performed to detect specific differences between groups.

\section{Results}

A total of 32 participants were included in the baseline measurement, after which 18 were exposed to the alcohol scrub (AS) intervention, 12 had a scrub nurse (SN) assist them during scrubbing, and 12 completed a self-wash (SW) with taps adjusted to open and close more easily.

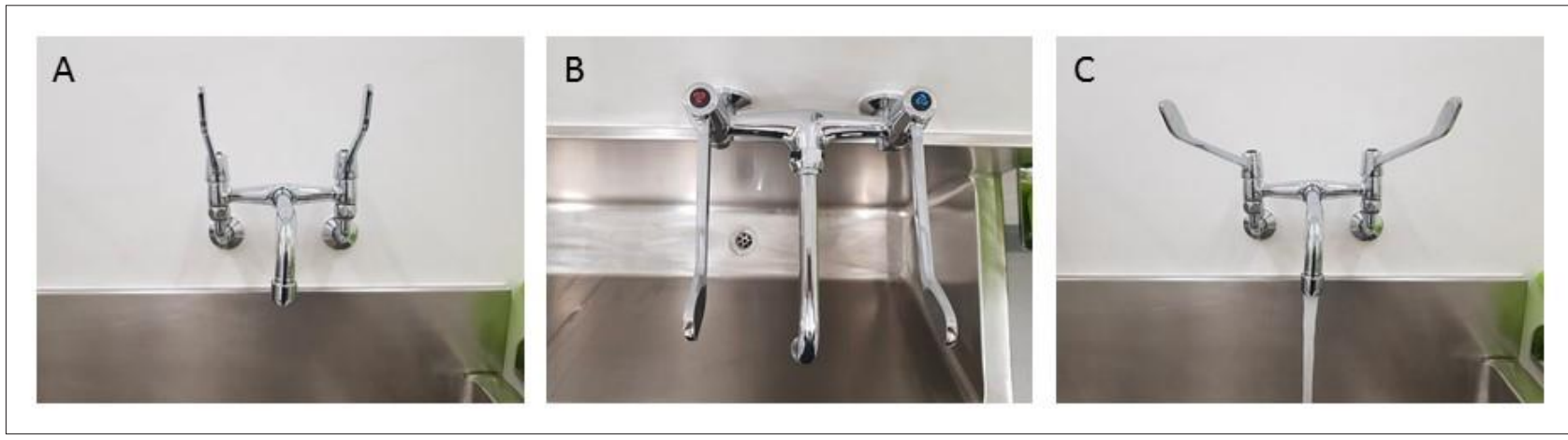

Fig. 1. Adjusted tap levers in the closed position (A and B) and the open position (C). 
Mean water consumption, wash time and flow rate were higher for the CON group than for the intervention groups (Table 1).

\section{Post hoc analyses}

Significant differences between the CON group and all three intervention groups independently were observed for the amount of water used per scrub $(p<0.001, p<0.001$ and $p<0.001$, respectively) (Fig. 2). Additionally, the AS intervention used significantly less water per scrub than the $\mathrm{SN}$ intervention $(p=0.033)$. There was no significant difference between the AS and SW interventions (Fig. 2).

The amount of time spent per scrub was significantly less in the AS group than in the SN group $(p=0.002)$, the SW group $(p<0.001)$ and the CON group $(p<0.001)$ (Fig. 3$)$.

Significant differences in tap flow rate $(\mathrm{L} / \mathrm{min})$ were observed between the CON group and the SN group $(p=0.005)$ and the SW group ( $p<0.001)$ (Fig. 4).

\section{Discussion}

The objective of this study was to compare water use during surgical hand preparation before and after specific water-saving interventions, to determine whether these changes can save water during surgical hand preparation in theatre. The first main finding of this study was that, compared with current practice at our institution, water consumption can be significantly reduced by employing one of three easy-to-implement interventions: (i) use of an AS; (ii) having an assistant to open and close taps during scrubbing; and (iii) an adjusted angle of the standard elbow tap to enable the surgeon to open and close the tap easily.

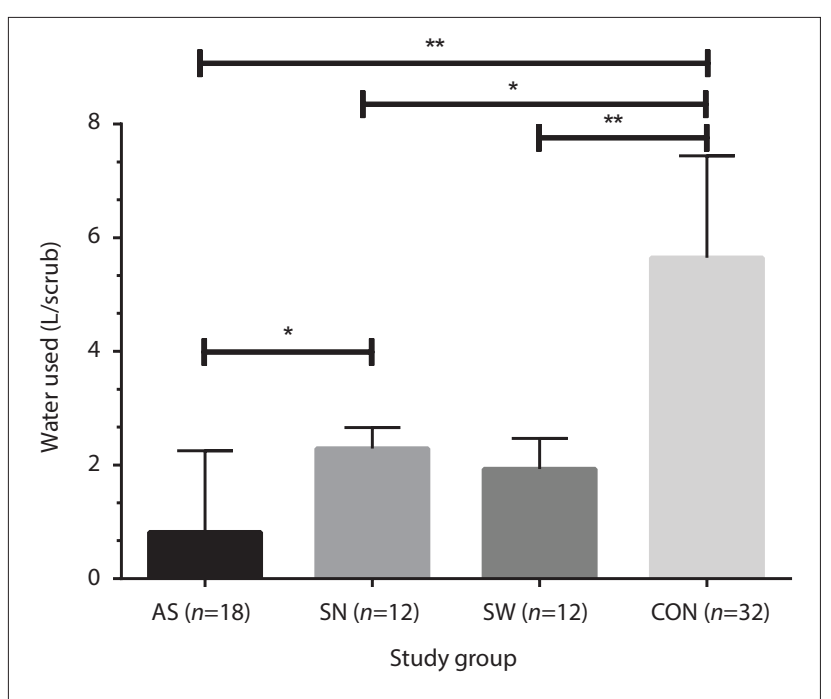

Fig. 2. Difference in the amount of water used per scrub between the groups. ( $A S$ = alcohol scrub; $S N=$ scrub nurse; $S W=$ self-wash with adjusted taps; CON $=$ control; ${ }^{*}$ post hoc statistical tests indicating $\mathrm{p}<0.05 ;{ }^{* *}$ post hoc statistical tests indicating $\mathrm{p}<0.001$.)
Water scarcity is becoming more prevalent across the world. The World Economic Forum highlighted the water crisis as the most significant risk worldwide in terms of potential impact in 2015, and further listed it as being in the top 10 risks in terms of likelihood of occurring. ${ }^{[9]}$ Water scarcity is experienced by as much as two-thirds of the world's population during at least part of a given year, ${ }^{[10]}$ and despite widespread perception that this is a problem mostly faced in developing countries, developed-world settings are increasingly experiencing decreased availability of clean water. ${ }^{[10,1]}$ In

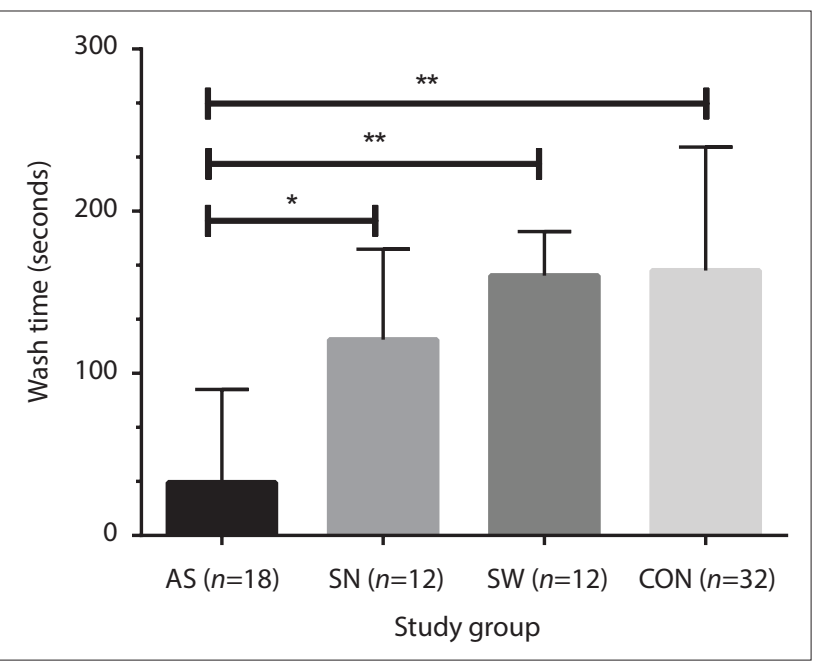

Fig. 3. Difference in mean wash time between the groups. (AS = alcohol scrub; $S N=$ scrub nurse; $S W=$ self-wash with adjusted taps; $C O N=$ control; ${ }^{*}$ post hoc statistical test indicating $\mathrm{p}<0.05 ;{ }^{* *}$ post hoc statistical tests indicating $\mathrm{p}<0.001$.)

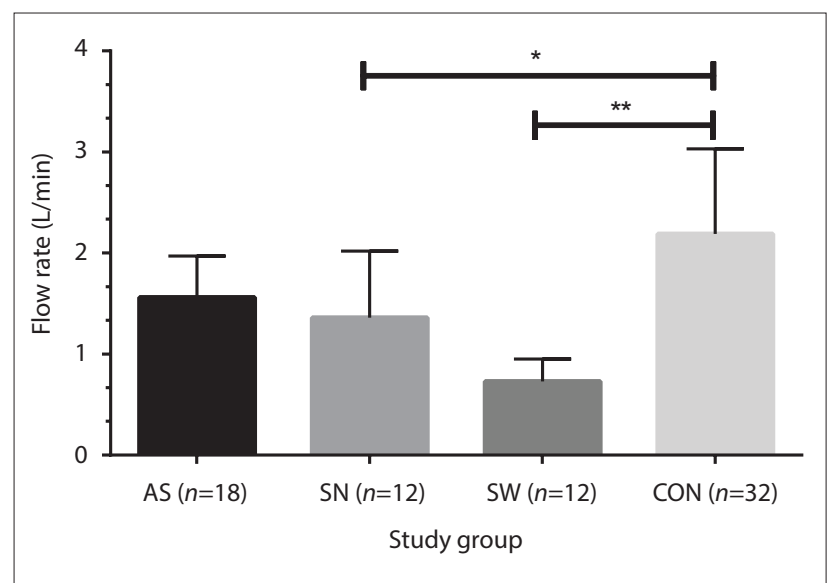

Fig. 4. Difference in mean water flow rate between the groups. $(A S=$ alcohol scrub; $S N=$ scrub nurse; $S W=$ self-wash with adjusted taps; $C O N=$ control; ${ }^{*}$ post hoc statistical test indicating $\mathrm{p}<0.05 ;{ }^{*}$ post hoc statistical test indicating $\mathrm{p}<0.001$.)

Table 1. Overview of the mean amount of water used, wash time during scrubbing and flow rate of water*

\begin{tabular}{|c|c|c|c|c|c|}
\hline & \multirow[b]{2}{*}{ Control $(n=32)$} & \multicolumn{3}{|c|}{ Intervention groups } & \multirow[b]{2}{*}{$p$-value } \\
\hline & & AS $(n=18)$ & SN $(n=12)$ & SW $(n=12)$ & \\
\hline Water used (L/scrub) & $5.65(1.79)$ & $0.82(1.43)$ & $2.29(0.37)$ & $1.93(0.54)$ & $<0.001$ \\
\hline Wash time (s) & $163.5(76.1)$ & $32.8(57.3)$ & $120.8(55.8)$ & $160.3(27.07)$ & $<0.001$ \\
\hline Flow rate $(\mathrm{L} / \mathrm{min})$ & $2.19(0.84)$ & $1.56(0.41)$ & $1.36(0.66)$ & $0.73(0.22)$ & $<0.001$ \\
\hline
\end{tabular}


SA, emphasis has been placed on encouraging the consumer to save water, both in the home and at the workplace. Although the use of clean water in operating theatres cannot be negotiated, water-saving mechanisms to reduce waste can certainly be explored.

The present study investigated three potential water-saving mechanisms that can easily be implemented in operating theatres that still use the traditional 'elbow tap' basins, which allow the surgeon to easily close, but not easily open, a tap during the scrubbing process. The first intervention employed the use of an AS. This method is widely used in operating theatres across the world and is endorsed by the WHO. ${ }^{[4]}$ Using an AS has previously been shown to lead to significant water savings, in addition to potentially being more effective than the traditional soap-and-water scrub. ${ }^{[12]}$ This method makes use of the AS between surgical cases, after an initial soap-and-water scrub at the start of the operating day. In the present study, this method yielded the lowest water use of all the interventions, with only $0.82 \mathrm{~L}$ water used on average per scrub. The total time spent scrubbing was also less compared with the baseline and other intervention groups. However, it should be noted that this method is in fact routinely available in our institution, yet surgeons prefer to use the traditional soap-and-water scrubbing method, as is evident from the difference between the baseline and the AS intervention group. Since surgeons could use their preferred method of scrubbing, including an AS, in the baseline measurements, it is clear that there was not wide support for this method among the participants included in the study. Although it uses least water, and there is evidence of improved efficacy, ${ }^{[12]}$ implementation of this method as routine practice in our institution may therefore not be feasible at present.

The second intervention we investigated was the use of an assistant to open and close the taps during the scrubbing process (SN). A previous study reported that, during conventional scrubbing, hands are only under running water for $29.2 \%$ of the total scrubbing process. ${ }^{[13]}$ Having a tap running during the remaining $70.8 \%$ of scrubbing time is therefore unnecessary wastage. That study reported higher water use per scrub and much higher flow rates $(20 \mathrm{~L} / \mathrm{min})$ than the CON group in the present study $(5 \mathrm{~L} / \mathrm{min})$. We speculate that this may be because sensitisation of surgeons to the ongoing drought in the Western Cape had already altered their behaviour. However, we did find similar (69\%) water wastage during scrubbing when calculating the difference in water use between the CON group and the SW group. This reduction was also observed in the SN group. Controlling water flow during hand scrubbing, by the surgeon or by an assistant, can therefore lead to substantial savings. However, each institution should be evaluated to determine whether the staffing situation would allow for an assistant to open and close the tap: as often experienced in resource-limited settings, it is likely that such an individual would not readily be available. In our institution, where between 50 and 100 surgical procedures take place daily in $\sim 20$ theatres, the practicality of this intervention could also be questioned.

The elbow-operated tap is conventionally used to prevent recontamination of hands during surgical hand preparation, and ideally these taps should be turned on and off using the elbow. ${ }^{[14]}$ However, a recent study in the UK highlighted the fact that elbow taps are often incorrectly installed and staff members routinely use their hands to operate them, leading to a high rate of recontamination. ${ }^{[14]}$ In the present study, the angle of the elbow-operated taps was changed to $45^{\circ}$, which made it easier for surgeons to open and close the taps with their elbows during scrubbing procedures. Surgeons making use of this method (SW) used a mean (standard deviation) of 1.93 (0.54) L water per scrub, nearly one-third of amount used at baseline. This amount was not significantly different from the amount of water used during the AS intervention, which highlights that a substantial amount of water can be saved by using this method. There was no difference between the three soap-and-water methods in the amount of time spent scrubbing, while the AS process was, as can be expected, significantly shorter.

Marinoski et al. ${ }^{[15]}$ reported that using water-efficient appliances alone can make a substantial difference in the amount of water saved during daily use. Although successful recycling mechanisms can increase water saving, the present study highlights that substantial savings can be made without the need for additional infrastructure. By extrapolating the use per scrub in our study to the entire hospital over the period of a year, a staggering $180000 \mathrm{~L}$ can be saved by implementing a simple, yet effective change in all hand wash basins at $\mathrm{TBH}$.

\section{Study limitations}

There are several limitations to this study. The baseline and intervention measurements were all taken over a short period of time, with a small number of surgeons participating per intervention. Additionally, a Hawthorne effect, where participants modified their behaviour based on the fact that they were being observed, was probably present even though participants were reminded not to deviate from their usual scrubbing procedures. However, we believe that this effect does not necessarily influence the conclusions that should be drawn from this study, since (i) the Hawthorne effect would have been present during the baseline measurement as well as the intervention components, and (ii) the focus of this study was on the robust differences in water use between the different methods, rather than the exact amount of water used. Finally, we did not consider the cost implications of the different interventions.

\section{Conclusions}

We believe that water use during surgical hand preparation can easily be decreased by implementing easy and effective interventions. Surgeon buy-in is important to ensure compliance. Ensuring that elbow-operated taps are correctly installed, for ease of opening and closing thereof, would equate to substantial savings over time.

Declaration. None.

Acknowledgements. The authors thank the participants who made this work possible.

Author contributions. The authors confirm that all authors made substantial contributions to all of the following: (i) conception and design of the study, acquisition of data, and analysis and interpretation of data; (ii) drafting the article and revising it critically for important intellectual content; (iii) final approval of the version to be submitted; and (iv) sound scientific research practice. They declare that this submission is in accordance with the principles laid down by the Responsible Research Publication Position Statements as developed at the 2nd World Conference on Research Integrity in Singapore, 21 - 24 July 2010.

Funding. None.

Conflicts of interest. None.

1. Department of Corporate Governance, South Africa. Reclassification of provincial disasters as a national disaster. National Gazette No. 41439, 13 February 2018, Vol. 632. https://www.greengazette. co.za/documents/national-gazette-41439-of-13-february-2018-vol-632 20180213-GGN-41439.pdf\# (accessed 31 January 2019)

2. City of Tshwane, South Africa. High water consumption. http://www.tshwane govza/sites/

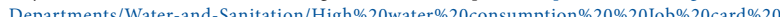


3. Price G. Water Conservation Guideline. Greening Durban 2010. eThekwini Municipality, October 2009. http://www.durban.gov.za/City_Services/development_planning_management/environmental_planning http://www.durban.gov.Za/City_Services/development_planning_management/environmental_
climate_protection/Publications/Documents/GG_Water_Guide.pdf (accessed 31 January 2019).

4. World Health Organization. WHO Guidelines on Hand Hygiene in Health Care. Geneva: WHO, 2009 World Health Organization. WHO Guidelines on Hand Hygiene in Health Care. Geneva: WHO, 2009.
https://apps.who.int/iris/bitstream/handle/10665/44102/9789241597906_eng.pdf;jsessionid=C522E3 https://apps.who.int/iris/bitstream/handle/10665/44102/9789241597906
14700EF4742EEDF936DFDB2E99? sequence=1 (accessed 29 June 2017).

5. Hubner N-O, Kampf G, Loffler H, Kramer A. Effect of a 1 min hand wash on the bactericidal efficacy of consecutive surgical hand disinfection with standard alcohols and on skin hydration. Int J Hyg Environ Health 2006;209(3):285-291. https://doi.org/10.1016/j.ijheh.2006.01.002

6. Tanner J, Dumville JC, Norman G, Fortnam M. Surgical hand antisepsis to reduce surgical site infection. Cochrane Database Syst Rev 2016, Issue 1. Art. No.: CD004288. https://doi.org/10.1002/14651858. CD004288.pub3

7. Parienti JJ, Thibon P, Heller R, et al. Hand-rubbing with an aqueous alcoholic solution vs traditional surgical hand-scrubbing and 30-day surgical site infection rates: A randomized equivalence study. JAMA 2002;288(6):722-727. https://doi.org/10.1001/jama.288.6.722

8. Widmer AF. Surgical hand hygiene: Scrub or rub? J Hosp Infect 2013;83(Suppl 1):S35-S39. https://doi org/10.1016/S0195-6701(13)60008-0

9. World Economic Forum. Global Risks 2015. 10th edition. Geneva: World Economic Forum, 2015. http:// www3.weforum.org/docs/WEF_Global_Risks_2015_Report15.pdf (accessed 11 February 2019).
10. Mekonnen MM, Hoekstra AY. Four billion people facing severe water scarcity. Sci Adv 2016;2(2):e1500323. https://doi.org/10.1126/sciadv.1500323

11. Fuller AC, Harhay MO. Population growth, climate change and water scarcity in the southwestern United States. Am J Environ Sci 2011;6(3):249-252. https://doi.org/10.3844/ajessp.2010.249.252

12. Jehle K, Jarrett N, Matthews S. Clean and green: Saving water in the operating theatre. Ann R Coll Surg Engl 2008;90(1):22-24. https://doi.org/10.1308/003588408X242277

13. Ahmed A. Surgical hand scrub: Lots of water wasted. Ann Afr Med 2007:6(1):31-33. https://doi org $/ 10.4103 / 1596-3519.55733$

14. Weinbren M, Bree L, Sleigh S, Griffiths M. Giving the tap the elbow? An observational study. J Hos Infect 2017;96(4):328-330. https://doi.org/10.1016/j.jhin.2017.05.009

15. Marinoski AK, Rupp RF, Ghisiw E. Environmental benefit analysis of strategies for potable wate savings in residential buildings. J Environ Manage 2018;206:28-39. https://doi.org/10.1016/j. jenvman.2017.10.004

Accepted 30 September 2019. 